\title{
A Method for Interval-valued Intuitionistic Fuzzy Multiple Attribute Decision Making with Incomplete Weight Information
}

\author{
Guiwu Wei ${ }^{1}$ Wende $\mathbf{Y i}^{2}$ \\ ${ }^{1}$ Department of Economy and Management, Chongqing University of Arts and Sciences, Chongqing 402160, \\ P.R.China \\ ${ }^{2}$ Department of Mathematics and Computer Science, Chongqing University of Arts and Sciences, Chongqing \\ 402160, P.R.China
}

\begin{abstract}
With respect to multiple attribute decision making problems with interval-valued intuitionistic fuzzy information, some operational laws of interval-valued intuitionistic fuzzy numbers, score function and accuracy function of interval-valued intuitionistic fuzzy numbers are introduced. An optimization model based on the ideal solution and min-max operator, by which the attribute weights can be determined, is established. We utilize the interval-valued intuitionistic fuzzy weighted arithmetic averaging (IIWAA) operator to aggregate the interval-valued intuitionistic fuzzy information corresponding to each alternative, and then rank the alternatives and select the most desirable one(s) according to the score function and accuracy function. Finally, an illustrative example is given to verify the developed approach and to demonstrate its practicality and effectiveness.
\end{abstract}

Keywords: Interval-valued intuitionistic fuzzy numbers, Operational laws, Interval-valued intuitionistic fuzzy weighted arithmetic averaging (IIWAA) operator, Incomplete weight

\section{Introduction}

Atanassov [1,2] introduced the concept of intuitionistic fuzzy set(IFS), which is a generalization of the concept of fuzzy set [3]. The intuitionistic fuzzy set has received more and more attention since its appearance. Gau and Buehrer [4] introduced the concept of vague set. But Bustince and Burillo [5] showed that vague sets are intuitionistic fuzzy sets. In [6], Xu developed some geometric aggregation operators, such as the intuitionistic fuzzy weighted geometric (IFWG) operator, the intuitionistic fuzzy ordered weighted geometric (IFOWG) operator, and the intuitionistic fuzzy hybrid geometric (IFHG) operator and gave an application of the IFHG operator to multiple attribute group decision making with intuitionistic fuzzy information. In [7], Xu developed some arithmetic aggregation operators, such as the intuitionistic fuzzy weighted averaging (IFWA) operator, the intuitionistic fuzzy ordered weighted averaging (IFOWA) operator, and the intuitionistic fuzzy hybrid aggregation(IFHA) operator. Later, Atanassov and Gargov [8-9] further introduced the interval-valued intuitionistic fuzzy set (IVIFS), which is a generalization of the IFS. The fundamental characteristic of the IVIFS is that the values of its membership function and nonmembership function are intervals rather than exact numbers. In [10], Xu developed some arithmetic aggregation operators, such as the interval-valued intuitionistic fuzzy weighted averaging (IIFWA) operator, the interval-valued intuitionistic fuzzy ordered weighted averaging (IIFOWA) operator, and the interval-valued intuitionistic fuzzy hybrid aggregation (IIFHA) operator and gave an application of the IIFHA operator to multiple attribute group decision making with interval-valued intuitionistic fuzzy information. In [11], $\mathrm{Xu}$ developed some geometric aggregation operators, such as the interval-valued intuitionistic fuzzy weighted geometric aggregation (IIFWGA) operator and the interval-valued intuitionistic fuzzy geometric aggregation (IIFGA) operator and gave an application of the IIFWGA and IIFGA operators to multiple attribute group decision making with interval-valued intuitionistic fuzzy information.

Multiple attribute decision making (MADM) has been one of the fastest growing areas during the last decades depending on the changings in the business sector. Decision maker(s) need a decision aid to decide between the alternatives and mainly excel less preferable alternatives fast. Especially in the last years, where computer usage has increased significantly, the application of MADM methods has considerably become easier for the users. In the recent years, MADM has received a great deal of attention from researchers in many disciplines [12-18].

In the process of MADM with interval-valued intuitionistic fuzzy information, sometimes, the attribute values take the form of interval-valued intuitionistic fuzzy numbers, and the information about attribute weights is incompletely known because of 
time pressure, lack of knowledge or data, and the expert's limited expertise about the problem domain. All of the above methods, however, will be unsuitable for dealing with such situations. Therefore, it is necessary to pay attention to this issue. The aim of this paper is to develop a method, based on the ideal solution and min-max operator, to overcome this limitation.

The remainder of this paper is set out as follows. In the next section, we introduce some basic concepts related to intuitionistic fuzzy sets and interval-valued intuitionistic fuzzy sets. In Section 3 we introduce the MADM problem with interval-valued intuitionistic fuzzy information, in which the information about attribute weights is incompletely known, and the attribute values take the form of interval-valued intuitionistic fuzzy numbers, An optimization model based on the ideal solution and min-max operator, by which the attribute weights can be determined, is established. We utilize the interval-valued intuitionistic fuzzy weighted arithmetic averaging (IIWAA) operator to aggregate the interval-valued intuitionistic fuzzy information corresponding to each alternative, and then rank the alternatives and select the most desirable one(s) according to the score function and accuracy function. In Section 4, an illustrative example is pointed out. In Section 5 we conclude the paper and give some remarks.

\section{Preliminaries}

In the following, we introduce some basic concepts related to intuitionistic fuzzy sets and interval-valued intuitionistic fuzzy sets.

Definition 1. Let $X$ be a universe of discourse, then a fuzzy set is defined as:

$$
A=\left\{\left\langle x, \mu_{A}(x)\right\rangle \mid x \in X\right\}
$$

Which is characterized by a membership function $\mu_{A}: X \rightarrow[0,1]$, where $\mu_{A}(x)$ denotes the degree of membership of the element $x$ to the set $A$ [3].

Atanassov extended the fuzzy set to the IFS, shown as follows:

Definition 2. An IFS $A$ in $X$ is given by

$$
A=\left\{\left\langle x, \mu_{A}(x), v_{A}(x)\right\rangle \mid x \in X\right\}
$$

Where $\mu_{A}: X \rightarrow[0,1]$ and $v_{A}: X \rightarrow[0,1]$, with the condition

$$
0 \leq \mu_{A}(x)+v_{A}(x) \leq 1, \forall x \in X
$$

The numbers $\mu_{A}(x)$ and $v_{A}(x)$ represent, respectively, the membership degree and nonmembership degree of the element $x$ to the set $A[1,2]$.
Definition 3. For each IFS $A$ in $X$, if

$$
\pi_{A}(x)=1-\mu_{A}(x)-v_{A}(x), \forall x \in X .
$$

Then $\pi_{A}(x)$ is called the degree of indeterminacy of $x$ to $A[1,2]$.

Definition 4. Let $X$ be a universe of discourse, An IVIFS $\tilde{A}$ over $X$ is an object having the form [8-9]:

$$
\tilde{A}=\left\{\left\langle x, \tilde{\mu}_{A}(x), \tilde{v}_{A}(x)\right\rangle \mid x \in X\right\}
$$

Where $\tilde{\mu}_{A}(x) \subset[0,1]$ and $\tilde{v}_{A}(x) \subset[0,1]$ are interval numbers, and

$$
0 \leq \sup \left(\tilde{\mu}_{A}(x)\right)+\sup \left(\tilde{v}_{A}(x)\right) \leq 1, \forall x \in X
$$

For convenience, let $\tilde{\mu}_{A}(x)=[a, b], \tilde{v}_{A}(x)=[c, d]$, so $\tilde{A}=([a, b],[c, d])$

Definition 5. Let $\tilde{a}=([a, b],[c, d])$ be an intervalvalued intuitionistic fuzzy number, a score function $S$ of an interval-valued intuitionistic fuzzy value can be represented as follows [10-11]:

$$
S(\tilde{a})=\frac{a-c+b-d}{2}, S(\tilde{a}) \in[-1,1] .
$$

Definition 6. Let $\tilde{a}=([a, b],[c, d])$ be an intervalvalued intuitionistic fuzzy number, a accuracy function $H$ of an interval-valued intuitionistic fuzzy value can be represented as follows [10-11]:

$$
H(\tilde{a})=\frac{a+b+c+d}{2}, H(\tilde{a}) \in[0,1] .
$$

to evaluate the degree of accuracy of the intervalvalued intuitionistic fuzzy value $\tilde{a}=([a, b],[c, d])$, where $H(\tilde{a}) \in[0,1]$. The larger the value of $H(\tilde{a})$, the more the degree of accuracy of the interval-valued intuitionistic fuzzy value $\tilde{a}$.

As presented above, the score function $S$ and the accuracy function $H$ are, respectively, defined as the difference and the sum of the membership function $\tilde{\mu}_{A}(x)$ and the non-membership function $\tilde{v}_{A}(x)$. $\mathrm{Xu}$ showed that the relation between the score function $S$ and the accuracy function $H$ is similar to the relation between mean and variance in statistics. Based on the score function $S$ and the accuracy function $H$, in the following, $\mathrm{Xu}$ give an order relation between two intuitionistic fuzzy values, which is defined as follows:

Definition 7. Let $\tilde{a}_{1}=\left(\left[a_{1}, b_{1}\right],\left[c_{1}, d_{1}\right]\right)$ and
$\tilde{a}_{2}=\left(\left[a_{2}, b_{2}\right],\left[c_{2}, d_{2}\right]\right)$ be two interval-valued 
intuitionistic fuzzy values, $s\left(\tilde{a}_{1}\right)=\frac{a_{1}-c_{1}+b_{1}-d_{1}}{2}$ and $s\left(\tilde{a}_{2}\right)=\frac{a_{2}-c_{2}+b_{2}-d_{2}}{2}$ be the scores of $\tilde{a}$ and $\tilde{b}$, respectively, and let $H\left(\tilde{a}_{1}\right)=\frac{a_{1}+c_{1}+b_{1}+d_{1}}{2}$ and $H\left(\tilde{a}_{2}\right)=\frac{a_{2}+c_{2}+b_{2}+d_{2}}{2}$ be the accuracy degrees of $\tilde{a}$ and $\tilde{b}$, respectively, then if $S(\tilde{a})<S(\tilde{b})$, then $\tilde{a}$ is smaller than $\tilde{b}$, denoted by $\tilde{a}<\tilde{b}$; if $S(\tilde{a})=S(\tilde{b})$, then

(1) if $H(\tilde{a})=H(\tilde{b})$, then $\tilde{a}$ and $\tilde{b}$ represent the same information, denoted by $\tilde{a}=\tilde{b}$; (2) if $H(\tilde{a})<H(\tilde{b}), \tilde{a}$ is smaller than $\tilde{b}$, denoted by $\tilde{a}<\tilde{b}[10-11]$.

$$
\text { Definition 8. Let } \quad \tilde{a}_{j}=\left(\left[a_{j}, b_{j}\right],\left[c_{j}, d_{j}\right]\right)
$$
$(j=1,2, \cdots, n)$ be a collection of interval-valued intuitionistic fuzzy values, and let IIWAA: $Q^{n} \rightarrow Q$, if

$$
\begin{aligned}
& \operatorname{IIWAA}_{w}\left(\tilde{a}_{1}, \tilde{a}_{2}, \cdots, \tilde{a}_{n}\right)=\sum_{j=1}^{n} \omega_{j} \tilde{a}_{j} \\
& =\left(\left[1-\prod_{j=1}^{n}\left(1-a_{j}\right)^{\omega_{j}}, 1-\prod_{j=1}^{n}\left(1-b_{j}\right)^{\omega_{j}}\right],\left[\prod_{j=1}^{n} c_{j}^{\omega_{j}}, \prod_{j=1}^{n} d_{j}^{\omega_{j}}\right]\right)
\end{aligned}
$$

where $\omega=\left(\omega_{1}, \omega_{2}, \cdots, \omega_{n}\right)^{T}$ be the weight vector of $\tilde{a}_{j}(j=1,2, \cdots, n)$, and $\omega_{j}>0, \sum_{j=1}^{n} \omega_{j}=1$, then IIFWAA is called the interval-valued intuitionistic fuzzy weighted arithmetic aggregation (IIWAA) operator [10-11]. Especially, if $\omega=(1 / n, 1 / n, \cdots, 1 / n)^{T}$, then IIWAA operator is reduced to the intuitionistic fuzzy arithmetic aggregation (IIAA) operator, which is defined as follows[10-11]:

$$
\operatorname{IIA} A\left(\tilde{a}_{1}, \tilde{a}_{2}, \cdots, \tilde{a}_{n}\right)=\frac{1}{n} \sum_{j=1}^{n} \tilde{a}_{j}
$$

Definition 9. Let $\tilde{a}_{j}^{(1)}=\left(\left[a_{j}^{(1)}, b_{j}^{(1)}\right],\left[c_{j}^{(1)}, d_{j}^{(1)}\right]\right)$ $(j=1,2, \cdots, n)$ and $\tilde{a}_{j}^{(2)}=\left(\left[a_{j}^{(2)}, b_{j}^{(2)}\right],\left[c_{j}^{(2)}, d_{j}^{(2)}\right]\right)$ $(j=1,2, \cdots, n)$ be two collections of interval-valued intuitionistic fuzzy values, then the weighted hamming distance between $\tilde{a}_{j}^{(1)} \quad(j=1,2, \cdots, n) \quad$ and $\tilde{a}_{j}^{(2)}(j=1,2, \cdots, n)$ is defined as follows:

$d\left(\tilde{a}_{j}^{(1)}, \tilde{a}_{j}^{(2)}\right)$

$=\frac{1}{4} \sum_{j=1}^{n} w_{j}\left[\left|a_{j}^{(1)}-a_{j}^{(2)}\right|+\left|b_{j}^{(1)}-b_{j}^{(2)}\right|+\left|c_{j}^{(1)}-c_{j}^{(2)}\right|+\left|d_{j}^{(1)}-d_{j}^{(2)}\right|\right]$

where $w=\left(w_{1}, w_{2}, \cdots, w_{n}\right)^{T}$ is weight vector of $\tilde{a}_{j}^{(i)}(i=1,2),(j=1,2, \cdots, n)[19]$.

\section{Interval-valued intuitionistic fuzzy decision making problems with incomplete weight information}

The following assumptions or notations are used to represent the interval-valued intuitionistic fuzzy MADM problems with incomplete weight information: (1) The alternatives are known. Let $A=\left\{A_{1}, A_{2}, \cdots, A_{m}\right\}$ be a discrete set of alternatives; (2) The attributes are known. Let $G=\left\{G_{1}, G_{2}, \cdots, G_{n}\right\}$ be a set of attributes;

(3)The information about attribute weights is incompletely known. Let $w=\left(w_{1}, w_{2}, \cdots, w_{n}\right) \in H$ be the weight vector of attributes, where $w_{j} \geq 0, j=1,2, \cdots, n, \sum_{j=1}^{n} w_{j}=1, H$ is a set of the known weight information, which can be constructed by the following forms [20-23], for $i \neq j$ : Form 1. A weak ranking: $w_{i} \geq w_{j}$; Form 2. A strict ranking: $w_{i}-w_{j} \geq \alpha_{i}, \alpha_{i}>0 ;$;Form 3. A ranking of differences: $w_{i}-w_{j} \geq w_{k}-w_{l}$, for $j \neq k \neq l$;Form 4. A ranking with multiples: $w_{i} \geq \beta_{i} w_{j}, 0 \leq \beta_{i} \leq 1$; Form 5. An interval form: $\alpha_{i} \leq w_{i} \leq \alpha_{i}+\varepsilon_{i}, 0 \leq \alpha_{i}<\alpha_{i}+\varepsilon_{i} \leq 1$.

Suppose that $\tilde{R}=\left(\tilde{r}_{i j}\right)_{m \times n}=\left(\left[a_{i j}, b_{i j}\right],\left[c_{i j}, d_{i j}\right]\right)_{m \times n}$ is the interval-valued intuitionistic fuzzy decision matrix, where $\left[a_{i j}, b_{i j}\right]$ indicates the degree that the alternative $A_{i}$ satisfies the attribute $G_{j}$ given by the decision maker, $\left[c_{i j}, d_{i j}\right]$ indicates the degree that the 
alternative $A_{i}$ doesn't satisfy the attribute $G_{j}$ given by the decision maker, $\left[a_{i j}, b_{i j}\right] \subset[0,1]$, $\left[c_{i j}, d_{i j}\right] \subset[0,1], \quad b_{i j}+d_{i j} \leq 1, \quad i=1,2, \cdots, m$, $j=1,2, \cdots, n$.

Definition 10. Let $\tilde{R}=\left(\tilde{r}_{i j}\right)_{m \times n}=\left(\left[a_{i j}, b_{i j}\right],\left[c_{i j}, d_{i j}\right]\right)_{\operatorname{man}}$ be an interval-valued intuitionistic fuzzy decision matrix, $\tilde{r}_{i}=\left(\tilde{r}_{i 1}, \tilde{r}_{i 2}, \cdots, \tilde{r}_{i n}\right)$ be the vector of attribute values corresponding to the alternative $A_{i}, i=1,2, \cdots, m$, then we call

$$
\begin{aligned}
& \Delta\left(\tilde{r}_{i}\right)=\left(\left[a_{i}, b_{i}\right],\left[c_{i}, d_{i}\right]\right)=I I W A A_{w}\left(\tilde{r}_{i 1}, \tilde{r}_{i 2}, \cdots, \tilde{r}_{i n}\right) \\
& =\left(\left[1-\prod_{j=1}^{n}\left(1-a_{i j}\right)^{w_{j}}, 1-\prod_{j=1}^{n}\left(1-b_{i j}\right)^{w_{j}}\right],\left[\prod_{j=1}^{n} c_{i j}{ }^{w_{j}}, \prod_{j=1}^{n} d_{i j}{ }_{i j}{ }^{w_{j}}\right]\right) \\
& i \in M,
\end{aligned}
$$

the overall value of the alternative $A_{i}$, where $w=\left(w_{1}, w_{2}, \cdots, w_{n}\right)^{T}$ is the weight vector of attributes.

In the situation where the information about attribute weights is completely known, i.e., each attribute weight can be provided by the expert with crisp numerical value, we can weight each attribute value and aggregate all the weighted attribute values corresponding to each alternative into an overall one by using Eq. (9). Based on the overall attribute values $\Delta\left(\tilde{r}_{i}\right)$ of the alternatives $A_{i}(i=1,2, \cdots, m)$, we can rank all these alternatives and then select the most desirable one(s). The greater $\Delta\left(\tilde{r}_{i}\right)$, the better the alternative $A_{i}$ will be.

Definition 11. Let $\tilde{R}=\left(\tilde{r}_{i j}\right)_{m \times n}=\left(\left[a_{i j}, b_{i j}\right],\left[c_{i j}, d_{i j}\right]\right)_{m \times n}$ be an interval-valued intuitionistic fuzzy decision matrix, $\tilde{r}^{+}=\left(\left[a_{1}^{+}, b_{1}^{+}\right],\left[c_{1}^{+}, d_{1}^{+}\right],\left[a_{2}^{+}, b_{2}^{+}\right],\left[c_{2}^{+}, d_{2}^{+}\right], \cdots,\left[a_{n}^{+}, b_{n}^{+}\right],\left[c_{n}^{+}, d_{n}^{+}\right]\right)$ be the ideal point of attribute values, defined as follows

$$
\begin{array}{r}
\left(\left[a_{j}^{+}, b_{j}^{+}\right],\left[c_{j}^{+}, d_{j}^{+}\right]\right)=\left(\left[\max _{i} a_{i j}, \max _{i} b_{i j}\right],\left[\min _{i} c_{i j}, \min _{i} d_{i j}\right]\right) \\
, j \in 1,2, \cdots, n .
\end{array}
$$

In the real life, there always exist some differences between the vector of attribute values corresponding to ideal point and the vector of attribute values corresponding to the alternative $A_{i}(i=1,2, \cdots, m)$. By Definitions 9 , in what follows we define the weighted hamming distance $d\left(\tilde{r}_{i}, \tilde{r}^{+}\right)$between the vector of attribute values $\tilde{r}^{+}$of ideal point and the vector of attribute values $\tilde{r}_{i}$ corresponding to the alternative $A_{i}(i=1,2, \cdots, m)$ :

$$
\begin{aligned}
& D_{i}(w)=d\left(\tilde{r}_{i}, \tilde{r}^{+}\right) \\
& =\frac{1}{4} \sum_{j=1}^{n} w_{j}\left[\left|a_{i j}-a_{j}^{+}\right|+\left|b_{i j}-b_{j}^{+}\right|+\left|c_{i j}-c_{j}^{+}\right|+\left|d_{i j}-d_{j}^{+}\right|\right]
\end{aligned}
$$

Obviously, the smaller $D_{i}(w)$, the better the alternative $A_{i}$ will be. Thus, a reasonable weight vector $w^{*}=\left(w_{1}^{*}, w_{2}^{*}, \cdots, w_{n}^{*}\right)$ should be determined so as to make all the distances $D_{i}(w)$ $(i=1,2, \cdots, m)$ as smaller as possible, which means to minimize the following distance vector:

$$
D(w)=\left(D_{1}(w), D_{2}(w), \cdots, D_{m}(w)\right)
$$

under the condition $w \in H$, where $H$ is the set of the known weight information defined as in Section 3.

In order to do that, we establish the following multiple objective optimization model:

$(\mathrm{M}-1)$ minimize $D(w)=\left(D_{1}(w), D_{2}(w), \cdots, D_{m}(w)\right)$

$$
\text { subject to } w \in H
$$

We utilize the min-max operator proposed by Zimmermann and Zysco [24] to integrate all the differences $D_{i}(w) \quad(i=1,2, \cdots, m)$, i.e., we get a single-objective programming model:

(M-2) minimize $\lambda_{1}$

subject to: $D_{i}(w) \leq \lambda_{1}, \quad i=1,2, \cdots, m$

$w \in H$

where

$$
\lambda_{1}=\max _{i} D_{i}(w)
$$

By solving the model $(\mathrm{M}-2)$, we get the optimal solution $w^{*}=\left(w_{1}^{*}, w_{2}^{*}, \cdots, w_{n}^{*}\right)$, which can be used as the weight vector of attributes.

Based on the above models, we develop a practical method for solving the MADM problems, in which the information about attribute weights is incompletely known, and the attribute values take the form of interval-valued intuitionistic fuzzy information. The method involves the following steps: 
Step 1. Let $\tilde{R}=\left(\tilde{r}_{i j}\right)_{m \times n}$ be an interval-valued intuitionistic fuzzy decision matrix, where $\tilde{r}_{i j}=\left(\left[a_{i j}, b_{i j}\right],\left[c_{i j}, d_{i j}\right]\right)$, which is an attribute value, given by an expert, for the alternative $A_{i} \in A$ with respect to the attribute $G_{j} \in G, \tilde{r}_{i}=\left(\tilde{r}_{i 1}, \tilde{r}_{i 2}, \cdots, \tilde{r}_{i n}\right)$ be the vector of attribute values corresponding to the alternative $\quad A_{i} \quad, \quad i=1,2, \cdots, m$, $\tilde{r}^{+}=\left(\tilde{r}_{1}^{+}, \tilde{r}_{2}^{+}, \cdots, \tilde{r}_{n}^{+}\right)$be the ideal point of attribute values, defined as in Definition 11, $w=\left(w_{1}, w_{2}, \cdots, w_{n}\right) \in H$ be the weight vector of attributes, where $w_{j} \geq 0$, $j=1,2, \cdots, n, \sum_{j=1}^{n} w_{j}=1, H$ is a set of the known weight information, which can be constructed by the forms 1-5.

Step 2. If the information about the attribute weights is partly known, then we solve the model (M-2) to obtain the attribute weights.

Step 3. Utilize the weight vector $w^{*}=\left(w_{1}^{*}, w_{2}^{*}, \cdots, w_{n}^{*}\right)$ and by Eq. (9), we obtain the overall values $\Delta\left(\tilde{r}_{i}\right)$ of the alternatives $A_{i}(i=1,2, \cdots, m)$.

Step 4. calculate the scores $S\left(\Delta\left(\tilde{r}_{i}\right)\right)(i=1,2, \cdots, m)$ of the overall interval-valued intuitionistic fuzzy preference values $\Delta\left(\tilde{r}_{i}\right)(i=1,2, \cdots, m)$ to rank all the alternatives $A_{i}(i=1,2, \cdots, m)$ and then to select the best one(s) (if there is no difference between two scores $S\left(\Delta\left(\tilde{r}_{i}\right)\right)$ and $S\left(\Delta\left(\tilde{r}_{j}\right)\right)$, then we need to calculate the accuracy degrees $H\left(\Delta\left(\tilde{r}_{i}\right)\right)$ and $H\left(\Delta\left(\tilde{r}_{j}\right)\right)$ of the overall interval-valued intuitionistic fuzzy preference values $\Delta\left(\tilde{r}_{i}\right)$ and $\Delta\left(\tilde{r}_{j}\right)$, respectively, and then rank the alternatives $A_{i}$ and $A_{j}$ in accordance with the accuracy degrees $H\left(\Delta\left(\tilde{r}_{i}\right)\right)$ and $H\left(\Delta\left(\tilde{r}_{j}\right)\right)$.
Step 5. Rank all the alternatives $A_{i}(i=1,2, \cdots, m)$ and select the best one(s) in accordance with $S\left(\Delta\left(\tilde{r}_{i}\right)\right)$ and $H\left(\Delta\left(\tilde{r}_{i}\right)\right)(i=1,2, \cdots, m)$.

Step 6. End.

\section{Illustrative example}

Let us suppose there is an investment company, which wants to invest a sum of money in the best option (adapted from [25]). There is a panel with five possible alternatives to invest the money: (1) $\mathrm{A}_{1}$ is a car company; (2) $A_{2}$ is a food company; (3) $A_{3}$ is a computer company; (4) $\mathrm{A}_{4}$ is a arms company. The investment company must take a decision according to the following four attributes: (1) $G_{1}$ is the risk analysis; (2) $G_{2}$ is the growth analysis; (3) $G_{3}$ is the environmental impact analysis. The four possible alternatives $A_{i}(i=1,2,3,4)$ are to be evaluated using the interval-valued intuitionistic fuzzy information by the decision maker under the above three attributes, as listed in the following matrix.

$\tilde{R}=\left[\begin{array}{lll}([0.4,0.5],[0.3,0.4]) & ([0.4,0.6],[0.2,0.4]) & ([0.1,0.3],[0.5,0.6]) \\ ([0.6,0.7],[0.2,0.3]) & ([0.6,0.7],[0.2,0.3]) & ([0.4,0.7],[0.1,0.2]) \\ ([0.3,0.6],[0.3,0.4]) & ([0.5,0.6],[0.3,0.4]) & ([0.5,0.6],[0.1,0.3]) \\ ([0.7,0.8],[0.1,0.2]) & ([0.6,0.7],[0.1,0.3]) & ([0.3,0.4],[0.1,0.2])\end{array}\right]$

The information about the attribute weights is partly known and the known weight information is given as follows:

$$
\begin{aligned}
H=\{ & 0.2 \leq w_{1} \leq 0.35,0.15 \leq w_{2} \leq 0.25,0.25 \leq w_{3} \leq 0.45, \\
& \left.0.7 w_{1} \leq w_{3}, w_{j} \geq 0, j=1,2,3, \sum_{j=1}^{3} w_{j}=1\right\}
\end{aligned}
$$

Then, we utilize the approach developed to get the most desirable alternative(s).

Step 1. Utilize the model (M-2) to establish the following single-objective programming model: minimize $\lambda_{1}$

$$
\begin{array}{ll}
\text { s.t. } & 0.25 w_{1}+0.125 w_{2}+0.4 w_{3} \leq \lambda_{1} \\
& 0.1 w_{1}+0.025 w_{2}+0.025 w_{3} \leq \lambda_{1} \\
& 0.25 w_{1}+0.125 w_{2}+0.05 w_{3} \leq \lambda_{1} \\
& 0.125 w_{3} \leq \lambda_{1} \\
& w \in H, \sum_{j=1}^{3} w_{j}=1, w_{j} \geq 0, j=1,2,3 .
\end{array}
$$

Solving this model, we get the weight vector of attributes:

$$
w=\left(\begin{array}{lll}
0.3442 & 0.2500 & 0.4058
\end{array}\right)^{T}
$$


Step 2. Utilize the weight vector $w^{*}=\left(w_{1}^{*}, w_{2}^{*}, \cdots, w_{n}^{*}\right)$ and by Eq. (9), we obtain the overall values $\Delta\left(\tilde{r}_{i}\right)$ of the alternatives $A_{i}(i=1,2, \cdots, m)$.

$$
\begin{aligned}
& \Delta\left(\tilde{r}_{1}\right)=([0.2927,0.4579],[0.3335,0.4715]) \\
& \Delta\left(\tilde{r}_{2}\right)=([0.5285,0.7000],[0.1510,0.2545]) \\
& \Delta\left(\tilde{r}_{3}\right)=([0.4386,0.6000],[0.1921,0.3559]) \\
& \Delta\left(\tilde{r}_{4}\right)=([0.5453,0.6543],[0.1000,0.2213])
\end{aligned}
$$

Step 3. calculate the scores $S\left(\Delta\left(\tilde{r}_{i}\right)\right)(i=1,2, \cdots, m)$ of the overall interval-valued intuitionistic fuzzy preference values $\Delta\left(\tilde{r}_{i}\right)(i=1,2, \cdots, m)$

$$
\begin{aligned}
& S\left(\tilde{r}_{1}\right)=-0.0272, S\left(\tilde{r}_{2}\right)=0.4115 \\
& S\left(\tilde{r}_{3}\right)=0.2453, S\left(\tilde{r}_{4}\right)=0.4392
\end{aligned}
$$

Step 4. Rank all the alternatives $A_{i}(i=1,2,3,4)$ in accordance with the scores $S\left(\Delta\left(\tilde{r}_{i}\right)\right) \quad(i=1,2, \cdots ; m)$ of the overall interval-valued intuitionistic fuzzy preference values $\Delta\left(\tilde{r}_{i}\right)(i=1,2, \cdots ; m): A_{4} \succ A_{2} \succ A_{3} \succ A_{1}$, and thus the most desirable alternative is $A_{4}$.

\section{Conclusions}

In this paper, we have investigated the problem of MADM with incompletely known information on attribute weights to which the attribute values are given in terms of interval-valued intuitionistic fuzzy numbers. To determine the attribute weights, an optimization model based on the ideal solution and min-max operator, by which the attribute weights can be determined, is established. We utilize the intervalvalued intuitionistic fuzzy weighted arithmetic averaging (IIWAA) operator to aggregate the intervalvalued intuitionistic fuzzy information corresponding to each alternative, and then rank the alternatives and select the most desirable one(s) according to the score function and accuracy function. Finally, an illustrative example is given. In the future, we shall continue working in the application of the interval-valued intuitionistic fuzzy multiple attribute decision-making to other domains.

\section{References}

[1] K. Atanassov, Intuitionistic fuzzy sets. Fuzzy Sets and Systems, 20 :87-96,1986.
[2] K. Atanassov, More on intuitionistic fuzzy sets. Fuzzy Sets and Systems, 33:37- 46,1989.

[3] L. A. Zadeh, Fuzzy sets. Information and Control, 8:338- 356,1965.

[4] W.L. Gau and D. J. Buehrer, Vague sets.IEEE Transactions on Systems, Man and Cybernetics, 23 (2):610-614, 1993.

[5] H. Bustine and P. Burillo, Vague sets are intuitionistic fuzzy sets. Fuzzy Sets and Systems, 79:403-405,1996.

[6] Z.S. Xu and R. R. Yager, Some geometric aggregation operators based on intuitionistic fuzzy sets. International Journal of General System, 2006, in press.

[7] Z.S. Xu, Intuitionistic fuzzy aggregation operators. IEEE Transations on Fuzzy Systems, 2007,in press.

[8] K. Atanassov and G. Gargov, Interval-valued intuitionistic fuzzy sets. Fuzzy Sets and Systems, 31:343-349, 1989.

[9] K. Atanassov, Operators over interval-valued intuitionistic fuzzy sets. Fuzzy Sets and Systems, 64 (2):159-174,1994.

[10] Z.S. Xu and J. Chen, An approach to group decision making based on interval-valued intuitionistic judgment matrices. System EngineerTheory \& Practice,27(4):126-133, 2007.

[11] Z.S. Xu, Methods for aggregating interval-valued intuitionistic fuzzy information and their application to decision making. Control and decision, 22(2):215-219, 2007.

[12] T.L. Saaty, The Analytic Hierarchy Process, McGraw-Hill, New York, 1980.

[13] C.L. Hwang and K. Yoon, Multiple Attribute Decision Making, Springer, Verlag, Berlin, 1981.

[14] M. Zeeny, Multiple Criteria Decision Making, McGraw-Hill, New York, 1982.

[15]S. J. Chen and C. L. Hwang, Fuzzy Multiple Attribute Decision Making, Springer, Berlin, 1992.

[16] J.C. Fodor and M. Roubens, Fuzzy Preference Modeling and Multicriteria Decision Support, Kluwer, Dordrecht, 1994.

[17] R.R. Yager and J. Kacprzyk, The Ordered Weighted Averaging Operators: Theory and Applications, Kluwer, Boston, 1997.

[18] Z. S. Xu, Uncertain Multiple Attribute Decision Making: Methods and Applications, Tsinghua University Press, Beijing, 2004.

[19] Z.S. Xu, On similarity measures of intervalvalued intuitionistic fuzzy sets and their application to pattern regcgnitions. Journal of southeast University (English Edition), 23(1): 139-143, 2007.

[20] K.S. Park and S.H. Kim, Tools for interactive multi-attribute decision making with incompletely 
identified information. European Journal of Operational Research, 98:111-123, 1997.

[21] S.H. Kim, S.H. Choi and J.K. Kim, An interactive procedure for multiple attribute group decision making with incomplete information: rangebased approach. European Journal of Operational Research, 118:139-152, 1999.

[22] S.H. Kim and B.S. Ahn, Interactive group decision making procedure under incomplete information. European Journal of Operational Research, 116:498-507,1999.

[23] K.S. Park, Mathematical programming models for charactering dominance and potential optimality when multicriteria alternative values and weights are simultaneously incomplete. IEEE transactions on systems, man, and cybernetics-part A, Systems and Humans, 34:601-614, 2004.

[24] H.J. Zimmermann and P. Zysco, Latent connectives in human decision making. Fuzzy Sets and Systems, 4:37-51, 1980.

[25] F. Herrera and E. Herrera-Viedma, Linguistic decision analysis: steps for solving decision problems under linguistic information. Fuzzy Sets and Systems, 115:67-82,2000. 